\title{
Mobilisations contre le gangstérisme et production d'une communauté morale coercitive en Afrique du Sud
}

Anti-gangsterism Mobilizations and Production of a Coercive Moral Community in South Africa

\section{Laurent Fourchard}

\section{(2) OpenEdition}

Journals

\section{Édition électronique}

URL : http://journals.openedition.org/ejts/4692

DOI : $10.4000 /$ ejts.4692

ISSN : $1773-0546$

\section{Éditeur}

EJTS

\section{Référence électronique}

Laurent Fourchard, «Mobilisations contre le gangstérisme et production d'une communauté morale coercitive en Afrique du Sud », European Journal of Turkish Studies [En ligne], 15 | 2012, mis en ligne le 25 mars 2013, consulté le 16 février 2020. URL : http://journals.openedition.org/ejts/4692 ; DOI :

$10.4000 /$ ejts.4692

Ce document a été généré automatiquement le 16 février 2020.

(C) Some rights reserved / Creative Commons license 


\title{
Mobilisations contre le gangstérisme et production d'une communauté morale coercitive en Afrique du Sud
}

\author{
Anti-gangsterism Mobilizations and Production of a Coercive Moral Community \\ in South Africa
}

Laurent Fourchard

1 Depuis une dizaine d'années, une histoire sociale du gangstérisme a émergé en Afrique du Sud. Elle est venue confirmer qu'un milieu criminel violent et structuré était consubstantiel de l'histoire des townships, des mines et du système pénitentiaire d'Afrique du Sud'. Le terme de gangstérisme est retenu ici dans une acception plus large que celui des seules actions criminelles et/ou violentes perpétrées par des gangs structurés pour inclure le massif des délits mineurs et des violences ordinaires qui constituent aujourd'hui l'essentiel des actes rapportés à la police ${ }^{2}$. Bien que les premières soient certainement dissociables des seconds, les organisations mobilisées contre le gangstérisme associent le plus souvent les deux dans une vision singulière de l'ordre politique local, les luttes contre l'emprise des gangs dans les townships rejoignant une protestation plus large contre ce qui est perçu comme une dégénérescence sociale à combattre.

2 L'Afrique du Sud a une longue tradition d'organisations de lutte contre la criminalité, dont les appellations variées témoignent de l'origine a priori 'populaire': organisations d'autodéfense (self-policing), police des gens (people's police), police communautaire (community police), ou vigilantes et vigilantisme. Sans revenir ici sur le sens de chacun de ces termes, notons que ces mobilisations 'du bas' ne s'opposent pas systématiquement à des initiatives qui viendraient 'du haut', en l'occurrence de la police. Les relations de ces multiples organisations à l'Etat sont au contraire ambivalentes et historiquement contingentes: une même organisation peut être tolérée avant d'être interdite, une initiative peut venir de la police avant de se transformer en mobilisation populaire, 
d'autres mouvements encore peuvent être 'absorbés' par la police. En Afrique du Sud, l'historicité de ces organisations est en partie établie, ce qui n'est pas le cas pour la plupart des pays d'Afrique subsaharienne ${ }^{3}$. Les recherches montrent qu'il s'agit d'une histoire faite de cycles de mobilisation et de démobilisation, face à une police essentiellement répressive (Seekings 2001; Kynoch 2003: 298-327; Goodhew 1993: 447-470). Les études sur la période post-1994 insistent de leur côté sur les transformations à l'œuvre dans la police, soit du fait de sa nouvelle coopération avec les communautés dites historiquement défavorisées (Mistry 1997), soit en raison de l'importation de nouveaux modèles internationaux (Rauch 2006; Singh 2008) et de la multiplicité des arrangements privés/publics dans la gestion de la sécurité (Baker 2008; Shearing, Berg 2006). Bien que les héritages de l'apartheid soient parfois mentionnés ${ }^{4}$, l'idée d'une police de l'apartheid politisée, militarisée, répressive et hostile aux populations non blanches (qui est la réalité policière des années 1980 au moment des affrontements avec les mouvements anti-apartheid) semble s'opposer à celle d'une police moins répressive, moins politisée, plus coopérative avec les populations 'historiquement défavorisées' depuis 1994. Cette histoire linéaire, guère contestable dans sa globalité, a l'inconvénient de faire l'économie d'une ambivalence des formes de répression et des formes de coopération avec les populations aussi bien pendant qu'après l'apartheid. Il faut donc étudier plus en détail les trajectoires historiques des mobilisations et des démobilisations sous l'effet des répressions policières sur la moyenne durée, sans exclure a priori la période post-apartheid (première partie). Il convient aussi de noter que la politisation de la police n'est pas moins forte depuis l'avènement d'un régime démocratique en Afrique du Sud. L'exemple de la province du Western Cape montre que les deux principaux partis rivaux (l'African National Congress, ANC, et la Democratic Alliance, DA) s'appuient sur ces mobilisations, en convoquant des registres historiques spécifiques pour consolider leur influence à l'échelle locale (deuxième partie). Enfin, la brutalité de la répression de la police d'apartheid a sans doute fait passer sous silence que la construction d'un ordre coercitif fut et reste le fait de citoyens bien ordinaires garants d'un ordre politique local, qui s'est construit principalement contre la jeunesse, hier comme aujourd'hui (troisième partie).

\section{Mobilisations contre le gangstérisme et répression politique}

Parmi les importantes mobilisations historiques contre le gangstérisme en Afrique du Sud, trois mouvements ont suscité un engouement sans précédent auprès des populations noires ou coloured ${ }^{5}$ des grandes villes: les gardes civiques (civic guards), un mouvement national qui s'est surtout implanté dans les quartiers noirs des villes du Rand dans les années 1940 et au début des années 1950, les Peacemakers, une organisation plus locale centrée sur quelques quartiers coloured du Cap dans les années 1970, et le Pagad (People Against Gangsterism and Drugs), un mouvement plus large qui opéra à l'échelle de la métropole du Cap dans la seconde moitié des années 1990. Il n'existe pas a priori de filiations historiques entre ces mouvements qui, par ailleurs, ne constituent que la partie la plus visible d'un ensemble d'organisations sur lesquelles nous sommes encore peu informés. 
4 Malgré leur trajectoire singulière, ces organisations ont en commun de lutter contre la violence, la drogue et une jeunesse supposée à la dérive, dangereuse et incontrôlable. Elles sont portées par des leaders politiques, religieux ou communautaires soutenus par une large base sociale, souvent territorialisée (à l'échelle du quartier) et composée pour l'essentiel de pères de famille. Le rapport de ces organisations à la police et plus généralement à l'Etat est sensiblement le même: ces mouvements ont été dans un premier temps tolérés, voire soutenus par les autorités locales et la police avant d'être interdits et réprimés pour des raisons politiques. Cette répression a eu pour effet sinon de les faire disparaître, tout du moins de réduire leur visibilité, de les atomiser, de les fragmenter, et de leur imposer de se recycler sous de nouvelles formes.

\section{Les gardes civiques : des origines policières et populaires}

Les gardes civiques représentent la plus large mobilisation contre le gangstérisme dans la première moitié du $\mathrm{xx}^{\mathrm{e}}$ siècle (Bénit et al. 2009: 115-141). Si, pour certains, leur origine est à rechercher du côté des patrouilles de quartier apparues dans plusieurs townships des villes du Rand dès la fin des années 1920 en réponse à la multiplication des gangs de tsotsis dans la région (Goodhew 1993: 447-470), elle semble plutôt liée à deux initiatives du gouvernement sud-africain lors de son entrée en guerre aux côtés des Alliés. La première est la réactivation par le gouvernement de la garde civique blanche - qui avait opéré de 1913 à 1919 à Johannesburg - par le recrutement de volontaires adultes de 30 à 50 ans patrouillant dans les quartiers du centre contre l'intrusion des natives', modèle qui se diffuse dans les quartiers noirs de Johannesburg pendant la guerre afin de combattre les tsotsis, figure locale du gangster. La seconde, émanant du ministère de l'Intérieur, est au début de 1940 le développement d'un Service de protection civil (CPS) dans lequel un ensemble de volontaires se voit confier la tâche d'assurer les mesures de précaution contre les bombardements (fabrication et entretien des abris anti-aériens, services aux blessés), mesures qui s'étendent rapidement à des opérations de routine policière. En 1942, le CPS compte 10000 volontaires répartis dans la plupart des grandes villes d'Afrique du Sud. Johannesburg et les villes du Rand concentrent 3000 volontaires blancs et 1000 volontaires noirs placés sous le commandement de la police sud-africaine. Le CPS sert ainsi de modèle au développement de la garde civique dans les townships pendant et après la guerre.

En 1947, à la suite du démantèlement des CPS par le gouvernement, certaines de ses branches se transforment en gardes civiques, agissant sans autorisation pendant plusieurs années. La reconnaissance légale de la garde civique devient alors l'une des principales revendications des leaders noirs pour lutter contre l'insécurité dans les townships. Ces demandes émanent aussi bien du département des Native Affairs que des résidents et des leaders politiques locaux.. En 1951, les membres noirs du Conseil de Johannesburg et plusieurs leaders de l'opposition dans le Rand pétitionnent contre le gouvernement au nom des 487000 Africains de Johannesburg (NASA 1951). Après avoir signalé que l'échec de la lutte contre la criminalité ne relève pas d'un accident mais d'un stratagème politique du gouvernement, ils proposent la mise en place d'un programme en dix points dans les villes et villages du Rand, incluant la reconnaissance de la garde civique, la création de cette garde dans les villages où elle n'existe pas encore et l'établissement de cours de justice présidées par des Africains (ibid.). Ces recommandations témoignent d'une relative cohésion des leaders noirs pour soutenir 
et diffuser des modèles sécuritaires alternatifs à la police sud-africaine dans l'ensemble du Transvaal. Il ne faudrait cependant pas opposer trop schématiquement une force locale noire à des forces de police blanches hostiles à toute coopération. Les principaux administrateurs du Département des affaires non-européennes (NEAD) de Johannesburg et les sous-officiers de police louent l'efficacité des gardes civiques et, dans certains quartiers (Western Areas township de Johannesburg), la police municipale accompagne même ces patrouilles.

7 Les liens supposés du mouvement avec les leaders de l'ANC et du Parti communiste, sa popularité au sein des townships inquiètent en revanche les responsables de la police sud-africaine et du Parti national, qui les présentent invariablement comme un bras armé du communisme (Kynoch 2005: 94-105). A Johannesburg, la mise sur pied d'une garde civique en août 1951 dans le quartier de Newclare, alors contrôlé par un gang sotho (connu sous le nom de Russians ou de Marashea), aboutit à de véritables batailles rangées entre les deux groupes en 1951-1952 (ibid.). En décembre 1952, le gouvernement décide de démanteler cette organisation et d'arrêter les récalcitrants au motif qu'ils « forçaient les habitants des townships à rejoindre leurs rangs et à payer des contributions obligatoires, qu'ils décourageaient les résidents à porter plainte à la police, et qu'ils infligeaient eux-mêmes des punitions corporelles aux populations locales $^{6} »$. Derrière ces raisons officielles, il est évident que le gouvernement est engagé dans une bataille pour l'hégémonie politique en Afrique du Sud et craint un mouvement trop populaire, trop large et trop proche de l'ANC. Gary Kynoch a montré que la police a pris prétexte des conflits répétés entre les Russians et les gardes civiques pour interdire les seconds tout en continuant à tolérer les premiers, considérés alors comme apolitiques (Kynoch 2005).

\section{L'atomisation des mobilisations: l'exemple des Peacemakers}

Le démantèlement des gardes civiles, suivi de l'interdiction de l'ANC et du Parti communiste en 1959, et la dislocation sociale engendrée par les politiques de déguerpissement massif des années 1950 et 1960 ont eu raison de ce premier mouvement. Le processus de contrôle social, qui fonctionne dans les premiers quartiers noirs, tend à se relâcher dans les nouveaux townships, alors que, simultanément, la clandestinité des activités militantes de l'ANC et du SACP et la suppression des organisations civiques ont créé un vide organisationnel (Seekings 2001; Suttner 2008). Aux larges mobilisations des années 1940 succédent pendant la période 1950-1976 des mobilisations très localisées. Ainsi, la plupart des organisations de gardes civiques ont pu survivre sporadiquement, parfois avec la tolérance des policiers locaux, pas toujours au fait de la liste des organisations interdites dans les townships. Elles ont alors pour périmètre d'action le quartier et leur espérance de vie se limite à quelques années, voire à quelques mois 7 . Cette période est également marquée par la prolifération de petites associations qui se réclament de la tradition et du monde rural et se prêtent ainsi aux projets gouvernementaux de retraditionnalisation de la société africaine (Badenhorst et al. 1997: 23 (1) 473-489). Ces organisations sont alors dirigées par des notabilités, membres des structures de gouvernement local qui prônent la nécessité d'un retour aux valeurs traditionnelles, au respect des aînés et à la rééducation de la jeunesse, au besoin par la trique. C'est le cas des comités de lutte contre la délinquance juvénile, des cours de parents (parent's courts), des Legkotla, conseils des aînés importés des campagnes dans les villes du Rand pour ressusciter les valeurs centrées sur le 
respect dû aux adultes (Glaser 2004). Ces associations ont de faibles capacités (au maximum une cinquantaine de membres) pour patrouiller leur quartier. En 1974, les deux plus importantes Legkotla de Soweto fusionnent pour former une plus grosse organisation, connue sous le nom de Makgotla, vite supplantée par les organisations étudiantes et lycéennes qui prennent le pouvoir à Soweto et dans les townships en 1976 (ibid.).

9 Le mouvement des Peacemakers du Cap, au milieu des années 1970, est un autre exemple significatif de cette période d'atomisation qui n'a pas encore retenu l'attention des chercheurs. Ce mouvement est né dans les Cape Flats, l'équivalent au Cap de Soweto, soit un ensemble de townships et de suburbs construits à partir du milieu des années 1960 pour loger les populations coloured et noires déguerpies des quartiers mixtes du centre, et notamment de District Six. Le quartier de Manenberg, lieu d'origine des Peacemakers, accueille principalement des populations ouvrières. A la différence des gardes civiques, les Peacemakers sont une initiative du bas qui n'a reçu ni l'appui de la police, ni celle des autorités locales ${ }^{8}$. Connu initialement sous le nom de 'Manenberg resident's movements (vigilantes)', le sobriquet de Peacemakers, donné par les gangsters, est vite adopté par les résidents et les membres du mouvement. L'ampleur et la rapidité de la mobilisation témoignent de sa popularité initiale : entre 1200 et 2000 membres recrutés en quelques mois selon les sources pour un quartier qui ne compte alors que 34000 habitants9 9 . Si cette initiative semble avoir été imitée dans d'autres quartiers du Cap, elle reste cependant largement identifiée au seul quartier de Manenberg. Les Peacemakers se sont réapproprié l'espace encaserné bâti par les ingénieurs de l'apartheid, notamment les immeubles locatifs bon marché à trois étages disposés autour d'une cour centrale (the courts). Ces cours deviennent leur principale unité d'opération; ils s'y regroupent le soir autour d'un feu, armés de bâtons, autant pour dissuader les gangs d'opérer que pour créer un espace de sociabilité familiale. A l'instar des gardes civiques, des confrontations entre les Peacemakers et certains gangs du Cap (les Mongrels, les Born Free Kids et les Mafias) ont pu mobiliser jusqu'à un millier de membres au cours d'affrontements violents relativement préparés ${ }^{10}$.

L'histoire du rapport des Peacemakers à l'Etat ressemble à celle des gardes civiques : une tolérance et une bienveillance initiales qui finissent en interdiction. Peu après sa formation, le mouvement demande et obtient une autorisation du ministère de l'Intérieur. Les autorités locales sont par ailleurs informées du fonctionnement des Peacemakers, et la police locale semble de son côté satisfaite de voir que ce système de surveillance contribue à faire baisser la criminalité dans le quartier ${ }^{11}$. Si son attitude commence à changer après la construction d'un commissariat de police en 1975, les officiers souhaitant désormais que les Peacemakers rejoignent la police en tant que volontaires, c'est le climat politique postérieur au soulèvement de Soweto en juin 1976 qui modifie la perception du gouvernement. Un temps, la police tente d'utiliser les Peacemakers pour réprimer le mouvement antiapartheid, mais comme une partie des Peacemakers ont finalement rejoint la mobilisation contre l'apartheid, les autorités décident de les interdire en 1979 (Kinnes 2000: 22). Le déménagement d'une partie des résidents de Manenberg dans une vaste zone résidentielle mi-ouvrière, mi-classe moyenne créée plus au sud du Cap, à Mitchell's Plain, permet à ce modèle de survivre et de renaître sous la forme des Neighbourhood Watch dans les années 1980, puis sous la forme plus radicale encore du Pagad dans les années 1990. 


\section{Des Neighbourhood Watch au Pagad}

11 Durant la période de 1976-1994, marquée par une lutte politique violente et intense entre le gouvernement et l'ensemble des mouvements antiapartheid, deux modèles policiers communautaires alternatifs émergent simultanément. L'un largement majoritaire à l'échelle du pays et dans les quartiers noirs des comités de rue, des comités de quartiers et des cours de justice populaire (People's Courts), l'autre plus marginal à l'échelle du pays des Neighbourhood Watch (NW) mais plus prégnant dans les quartiers coloured du Cap.

Dans les quartiers noirs, l'usage de la coercition, jusque-là monopole exclusif des aînés, passe alors à la jeunesse. Parés d'une nouvelle légitimité politique et rendus disponibles par les nombreux boycotts scolaires, les étudiants et lycéens prennent rapidement la relève des organisations des périodes précédentes (Legkotla, Makgotla) et s'y révèlent, semble-t-il, plus efficaces pour discipliner les gangs (Glaser 1998: 321). Pendant la révolte des townships de 1984-1986, quatre cents cours de justice populaire voient le jour, se substituant en partie aux structures de la police, des gouvernements locaux et des anciens leaders associatifs désormais discrédités. Elles établissent des patrouilles et des comités disciplinaires pour tenter de contrôler la violence ordinaire et prévenir les dérèglements sociaux (Seekings 2001: 91). La lutte contre le gangstérisme s'inscrit alors dans le combat plus large du combat de l'ANC et de ses alliés locaux contre l'apartheid.

Dans les quartiers coloured du Cap, des comités de rue et de quartier sont également mis en place, mais moins systématiquement et principalement pendant la période de mobilisation de l'United Democratic Front, l'UDF (1985-1990). Dans ces quartiers se diffuse parallèlement depuis les années 1980 une autre pratique policière, inspirée à la fois des Peacemakers et des NW, un modèle importé des Etats-Unis et qui repose sur la formation de patrouilles de volontaires à l'échelle du quartier. Le rapport à la police constitue dans les années 1980 l'une des principales différences entre les comités de rue et les NW. Les premiers ont été instaurés pour lutter contre le régime de l'apartheid, les seconds sont organisés parfois sans, parfois par la police, mais rarement contre elle. Ainsi, à partir de 1987, le commissaire central de Mitchell's Plain vante 'les dimensions non politiques, non raciales et non vigilante des NW' et propose d'en établir un dans chaque quartier de Mitchell's Plain ${ }^{12}$. A la fin des années 1980, les NW sont devenus le modèle dominant dans cette vaste zone résidentielle (avec des effectifs oscillant selon les périodes entre 1500 et 2500 membres).

14 Le Pagad, qui opère principalement dans la région du Western Cape entre 1995 et 2002, s'inscrit dans la tradition des NW, même si les études qui lui sont consacrées oublient parfois ces origines. Le mouvement naît en décembre 1995 de l'initiative d'une petite douzaine de NW de plusieurs quartiers coloured du Cap (Kensington, Salt River et Surrey Estate). Ses premières manifestations publiques témoignent d'une volonté d'exercer une pression populaire sur le gouvernement. En août 1996, l'exécution à son domicile de Rashaad Staggie, chef des Hard Livings Gangs, à la suite d'une marche organisée par le Pagad fait basculer le mouvement et marque le début d'une mobilisation sans précédent (rassemblements de plusieurs milliers de personnes dans les stades, dans les mosquées et dans la rue). Le mode opératoire de l'organisation se radicalise. Des manifestations organisées par le Pagad devant des maisons de dealers et de chefs de gang sont suivies de la remise aux intéressés d'un ultimatum exigeant la cessation de leurs activités criminelles dans les 24 heures: au-delà de ce délai, les gangsters et 
dealers seront laissés à la 'vindicte populaire'. Le Pagad revendique ainsi la suppression de trente gangsters en 1998 et 1999 (Kinnes 2000: 37). Nombre de maisons de revendeurs de drogue sont également incendiées pendant cette période.

S'il existe un relatif consensus sur l'origine du mouvement, la compréhension de ses transformations ultérieures est en revanche controversée. Pour la plupart des auteurs, le contrôle de l'organisation serait passé au cours de l'année 1996 à Qibla, un groupe radical islamique inspiré de la révolution iranienne, fondé au début des années 1980 pour lutter contre l'apartheid (Botha 2001; Galant et al. 1996; Shaw 2002: 98; Jensen 2008: 185-189; Baker 2002: 36; Kelly 2009: 126). Le Pagad se serait ainsi transformé en un mouvement ennemi de l'Etat mais aussi de l'ANC, Qibla étant lui-même allié au Pan African Congress, ennemi historique de l'ANC depuis sa scission en 1960. Cette mutation interne expliquerait également le changement de cibles de l'organisation (bombardements de commissariats de police, de propriétés de businessmen refusant de payer l'impôt de solidarité', de leaders musulmans hostiles au mouvement) et ses changements de méthode (utilisation de bombes artisanales contre des commerces ou des commissariats de police entre juin 1998 et août 2000).

Ces recherches reposent pour l'essentiel sur des sources étrangères à l'organisation elle-même, à l'exception d'une étude, la plus fouillée à ce jour (Dixon et al. 2001). Leurs auteurs réfutent le récit du passage d'un groupe populaire à un groupe islamiste, considérant au contraire qu'à partir du moment où les relations ont été rompues entre le gouvernement et l'organisation (fin 1996), le Pagad a été traité comme "un gang parmi d'autres ", obligé de se structurer et d'avancer caché. Le gouvernement n'a cessé d'associer ce mouvement au répertoire d'un islam militant fondamentaliste afin de le discréditer ${ }^{13}$. Or, dans la réalité, le lien entre Pagad et Qibla est difficile à établir, tandis que la justice n'a jamais réussi à condamner les membres du Pagad pour la série d'attentats qui a eu lieu au Cap contre les restaurants, les bars ou les centres commerciaux $^{14}$. Un haut responsable de la police du Western Cape et membre de l'ANC confirme que l'ennemi à abattre derrière le Pagad était bien Qibla et le PAC ${ }^{15}$. L'idée que le mouvement a été réprimé pour des raisons essentiellement politiques semble donc largement plausible.

17 Ainsi, par-delà la singularité historique de chacun de ces mouvements, l'histoire de ces mobilisations rend compte avant tout d'une histoire de la répression politique. Ce faisant, leur interdiction ne peut être comprise comme la manifestation d'un Etat désireux de conserver le monopole de la violence légitime. Au contraire, les gouvernements successifs comme les policiers sur le terrain se montrent initialement bienveillants à l'égard de mouvements qui assurent un relais dans les zones où la police est absente, et ferment les yeux ou tolèrent le recours fréquent à la violence inhérente à leur fonctionnement. En revanche, l'interdiction et la répression s'abattent sur eux à partir du moment où ils sont identifiés comme des organisations infiltrées par les ennemis politiques du parti au pouvoir (1951 pour les gardes civiques, 1979 pour les Peacemakers, 1997 pour le Pagad). Un répertoire de la stigmatisation banal mais efficace est alors convoqué (lutter contre des communistes hier, combattre les islamistes aujourd'hui) pour discréditer des organisations soupçonnées de nourrir un projet politique caché afin de mieux les dissocier des mobilisations populaires légitimes confrontées à la violence quotidienne dans les townships. La répression une fois engagée a pour effet sinon de faire disparaître ces mouvements, du moins de réduire leur visibilité, de les atomiser, de les fragmenter et de leur imposer de se recycler sous de 
nouvelles formes. Les mobilisations contre le gangstérisme constituent aujourd'hui dans la province du Cap une ressource politique d'autant plus centrale qu'elle est la seule province à pouvoir contester l'hégémonie politique de l'ANC à l'échelon national depuis 1994.

\section{Les ressources politiques de la police communautaire}

Pendant la période de transition (1990-1994), se pose la question de comment réformer la police d'apartheid, brutale, raciste et illégitime, en une police moins raciste, plus démocratique, plus responsable et plus respectueuse des droits de l'homme. L'objectif principal est de la réconcilier avec les populations 'historiquement défavorisées'. La police communautaire (community police) - un modèle américain et britannique des années 1980 exporté dans de nombreux pays anglophones dans les années 1990 - est vite identifiée en Afrique du Sud comme l'un des mécanismes permettant de restructurer et de légitimer la police. Ainsi, dans la nouvelle loi de 1995, le Forum de police communautaire (Community Police Forum, CPF) doit donner la parole aux communautés sur la manière dont elles veulent désormais être policées. En dépit d'un enthousiasme initial, il devient vite évident que la police communautaire ne peut faire face à un véritable problème national de violence criminelle (Burger 2007: 106-108). Par ailleurs, passée la période de transition politique et d'enthousiasme initial, les CPF deviennent des espaces accaparés par les représentants des partis politiques. Dans la province du Cap occidental, notamment, les CPF se révèlent une structure organisationnelle importante pour un ANC soucieux d'étendre son influence dans les circonscriptions coloured qui, depuis 1994, lui sont peu favorables.

La province du Cap apparaît à cet égard une province politiquement à part. La province comme la municipalité du Cap sont les seules à avoir connu plusieurs alternances: les principales forces politiques, l'ANC d'une part, le Parti national (de 1994 à 2000) puis l'Alliance démocratique, DA (depuis 2000), d'autre part, se livrent à chaque élection une bataille serrée pour le contrôle des principales municipalités et de la province ${ }^{16}$. Comme la DA ne recueille pratiquement aucune voix dans les quartiers noirs et que l'ANC a une implantation marginale dans les municipalités blanches, l'essentiel des efforts des militants des deux partis portent sur la majorité électorale de la province: les Coloured ${ }^{17}$.

\section{Des luttes politiques nationales à l'échelon local}

La compétition entre la DA et l'ANC dans la province du Western Cape a sensiblement politisé les actions sécuritaires au cours des dix dernières années. Deux importantes initiatives en témoignent: le programme provincial de l'ANC en matière de prise en charge de la sécurité des communautés depuis 2002, et la bataille entre la DA et l'ANC à propos des comités de rue et des NW à Mitchell's Plain depuis 2007. A la différence des répressions policières précédemment évoquées, ces exemples montrent que les partis politiques et la police sont des acteurs centraux de ces mobilisations et que les luttes d'influence nationale entre les deux principaux partis du pays ont des répercussions sur les formes de mobilisation contre le gangstérisme à l'échelle locale.

21 La lutte entre le Pagad, les gangs et la police à la fin des années 1990 a rapidement imposé au gouvernement de la province de développer un nouvel agenda sécuritaire. 
En 2001, Ibrahima Rasool, président de l'ANC pour la province du Western Cape et membre du gouvernement provincial de coalition (NP/ANC), déclare que la guerre contre les gangs est désormais l'une des priorités du gouvernement. Le ministre ANC de la Sécurité, Leonard Ramatlakane, lance à grand renfort de publicité un programme, People Orientated Problem Solving Policing Plan, plus connu sous le nom de Bambanani ${ }^{18}$ : le gouvernement s'engage à entraîner et à verser une indemnité à des centaines de 'volontaires' qui seront placés dans les trains, les gares, les écoles, les zones commerciales et sur les plages, tout en demandant aux communautés de patrouiller davantage leur quartier ${ }^{19}$. Cette première initiative est suivie en 2005 du programme 'Écoles plus sûres' (Safer Schools): parmi les 859 écoles, collèges et lycées de la province, 109 établissements dits 'sensibles' sont désignés pour profiter des services de volontaires indemnisés. La très inégale répartition des écoles bénéficiant de l'appui des Bambanani indique que cette sélection est davantage liée à la présence de relais locaux de l'ANC qu'à un classement rigoureux des collèges et des lycées les plus dangereux de la région, comme le révèle la différence de traitement entre Mitchell's Plain et Manenberg ${ }^{20}$. Par ailleurs, en proposant des indemnités journalières à des volontaires, ce programme contribue à populariser l'action du gouvernement dans des quartiers peu acquis à sa cause et devient de facto une initiative concurrente de la longue tradition des NW, service gratuit rendu au quartier par ses membres.

La réintroduction des comités de rue, symboles de la lutte de l'ANC contre l'apartheid, est un autre exemple significatif de la politisation des projets communautaires en matière de sécurité. Cette initiative est lancée en juin 2007 par Jeremy Vearey, nouveau directeur de la police de Mitchell's Plain, ancien membre de la branche militaire de l'ANC, compagnon de prison de Nelson Mandela à Robben Islands et chef du service de renseignement de la province à la fin des années $1990^{21}$. Ces nouveaux comités, à la différence de ceux des années 1980 , sont chapeautés par la police et visent initialement à mobiliser les mères de famille contre le trafic de drogue à l'échelle de leur rue. Leur mandat, rapporté par la presse locale à l'époque, paraît assez vague: 'être un bon voisin, un résident responsable pour la communauté, devenir l'œil et l'oreille de la police dans tous les quartiers ${ }^{22}$. Ces comités sont en réalité mis en place pour prévenir un retour du vigilantisme dans la région. En mai et juin 2007, plusieurs manifestations contre des résidences de dealers de drogue à Mitchell's Plain se sont achevées par l'incendie de leur maison, ravivant les inquiétudes d'un retour aux méthodes du Pagad. Les comités de rue sont créés pour éviter que ces manifestations ne dégénèrent en règlements de comptes violents. Leurs membres sont autorisés à s'installer en face de la résidence du dealer du quartier les soirs de fin de semaine pour surveiller les sorties et entrées de toute 'personne suspecte', l'objectif étant de limiter la circulation de drogue sans porter atteinte aux personnes et aux propriétés ${ }^{23}$. Dans cette opération, la force du leader de l'ANC est d'avoir su transformer une action sécuritaire pragmatique en une opération de marketing politique. De fait, l'initiative a été largement médiatisée par la police, qui signale qu'en trois mois 1646 comités de rue ont vu le jour et qu'ils sont devenus la plus grosse organisation luttant contre la criminalité au Cap ${ }^{24}$. Il semble que cette initiative ait d'ailleurs précédé l'appel des présidents Mbeki et Zuma à ressusciter les comités de rue pour combattre la criminalité dans tout le pays ${ }^{25}$.

Les initiatives mises en place dans les provinces du Limpopo et du KwaZulu-Natal ont suscité des protestations de l'Inkatha Freedom Party, qui les considérait en 2008 avant tout comme des structures de mobilisation de l'ANC ${ }^{26}$. Dans la province du Cap, cette impression est largement partagée dans les rangs de la DA, d'autant que Mitchell's 
Plain, à la différence de la province du Kwa Zulu-Natal, a peu de tradition de comités de rue. Ainsi, peu de temps après leur introduction, Hellen Zille, présidente de la DA et mairesse du Cap, accuse le directeur de la police de parrainer les comités de rue pour marginaliser les NW: d'après elle, 'il s'agit d'une stratégie de l'ANC pour retrouver ses soutiens perdus à Mitchell's Plain lors des élections de 200627'. De son côté, le Premier ministre du gouvernement provincial ANC accuse Helen Zille de soutenir une nouvelle association, le People Against Drugs, Liquor and Crime (Padlac), considérée par le gouvernement comme un mouvement vigilante proche du Pagad ${ }^{28}$. Le gouvernement reproche également à la municipalité son manque d'action dans la lutte contre la criminalitée ${ }^{29}$. Il paraît donc évident que les NW, les Bambanani et les comités de rue sont utilisés par les partis politiques pour maintenir ou étendre leur influence à l'échelle locale. Le responsable des programmes de police communautaire et membre de l'ANC depuis vingt ans le reconnaît volontiers ${ }^{30}$. Le registre mobilisé témoigne aussi de ces oppositions politiques, l'ANC parlant de comités de rue et de Bambanani là où la DA n'évoque que des NW.

\section{Au-delà des confrontations politiques}

Cette opposition est véhiculée par des leaders qui partagent une vision instrumentale de ces organisations. Localement, les comités de rue revêtent une autre signification. A l'échelle des présidents de CPF, des responsables de NW, des officiers de police, le discours public est tout autre: 'le soutien aux organisations communautaires vise à réduire la criminalité pas à faire de la politique', 'les comités de rue ne sont pas un programme de l'ANC', 'les NW n'appartiennent pas à DA'. Un tel discours dépolitisé est tenu dans les forums de police communautaire pour légitimer des opérations qui impliquent chaque week-end la mobilisation de milliers de citoyens pour surveiller les rues: il est au cœur du processus de bureaucratisation des initiatives communautaires (voir infra). De même, à la base, l'opposition entre les NW et les comités de rue n'est pas opératoire. Des dizaines de membres des NW ou des comités de rue interrogés ne revendiquent aucune appartenance à un parti politique et justifient leur engagement propos des dizaines de fois répétés - par le désir d'avoir un quartier plus sûr, débarrassé des vendeurs de drogue. L'inégale présence des NW et des comités de rue dans les différents secteurs de Mitchell's Plain ne relève pas d'une volonté des partis politiques de les soutenir, mais bien plutôt des conditions socio-économiques de chaque quartier, de la présence de leaders capables de mobiliser des troupes et du degré d'enracinement historique des NW en leur sein. Ainsi, l'un des premiers NW de Mitchell's Plain, celui de Rocklands créé en 1983, a connu une histoire cyclique, faite de mobilisations et de démobilisations, avant de se redynamiser à la fin de l'année 2008 grâce aux comités de rue. De nombreux membres de ces comités ont rejoint les NW pour ne pas limiter leur action à la seule surveillance de leur rue et participer à des patrouilles massives ( 80 membres) les soirs de week-end. Dans beaucoup d'autres quartiers, les collaborations entre les NW (plus historiques, plus institutionnalisés, plus équipés) et les comités de rue (plus récents, moins équipés et souvent spontanés) sont la norme. De même, les membres des NW sont souvent membres des Bambanani: comme le résume une femme de Manenberg travaillant depuis vingt-cinq ans à la sécurité de son quartier, les NW et les Bambanani font la même chose, seul le nom a changé ${ }^{31}$. 

nationales sont perçues par la base comme des dispositifs qui se recoupent, se chevauchent ou se recyclent sans vraiment s'opposer. On ne peut donc les réduire à des mobilisations strictement communautaires, à de simples initiatives policières ou à des instrumentalisations politiques: bien au contraire, leur caractéristique est plutôt d'être coproduite par la police, par les 'communautés' et par les partis. Si les rivalités politiciennes dans le contrôle des ressources de la sécurité communautaire se sont particulièrement exacerbées depuis une dizaine d'années dans cette région, elles participent in fine de la construction d'une autorité locale à l'échelle du township et sont partie prenante d'un processus de coercition et de bureaucratisation depuis bien plus longtemps à l'œuvre en Afrique du Sud.

\section{Des mobilisations entre coercition et bureaucratisation}

Une impression ressort lorsque l'on parcourt les Cape Flats la nuit: les jeunes sont singulièrement absents des patrouilles, dominées à une écrasante majorité par des pères et des mères de famille. L'appartenance au monde des adultes apparaît un élément structurant de ces formations policières et les jeunes en constituent les principales cibles. Cette fracture générationnelle renvoie à un rapport d'autorité historiquement construit entre aînés et cadets dont la jeunesse semble toujours prête à vouloir s'affranchir - jeunesse qu'il est nécessaire de rappeler à l'ordre, au besoin par des châtiments corporels. Rien de très original dans ce constat. Jean-François Bayart rappelle que le rapport d'autorité et de séniorité en Afrique passe volontiers par l'administration du fouet au moins au détriment des enfants, des adolescents ou des jeunes hommes, et que cette 'politique de la chicotte' est au cœur de la fabrique coercitive du politique du continent (Bayart 2008: 123-152). Par-delà cette histoire commune, qu'il reste à faire émerger pays par pays, deux singularités historiques sudafricaines sont à retenir : les politiques d'enfermements massifs du gouvernement sudafricain n'ont pas eu d'équivalent sur le continent, alors que les punitions corporelles sont demeurées au centre de ses institutions pénales jusqu'au début des années $1990^{32}$. Par ailleurs, le niveau de violences liées à la criminalité en Afrique du Sud est également probablement exceptionnel à l'échelle du continent ${ }^{33}$.

\section{La politique de la chicotte}

27 Les châtiments corporels ont été un moyen commun de discipliner la jeunesse sous le régime de l'apartheid, mais il paraît évident que cette pratique s'enracine dans une histoire plus longue, celle de la flagellation, au cœur du rapport maître-esclave jusqu'aux années 1840 dans la colonie du Cap, et qui perdure jusqu'à la fin du XIX siècle dans les Républiques boers de la frontière (Penn 1999: 79; Dooling 2007: 84-89). Les châtiments sont par la suite utilisés, comme dans beaucoup d'autres colonies d'Afrique, comme un moyen de mobiliser la main-d'œuvre et de discipliner une jeunesse récalcitrante à la campagne comme à la ville. Ils s'accentuent avec le régime de l'apartheid: dans l'esprit des criminologues du Parti nationaliste, un meilleur contrôle des Noirs dans les villes et des châtiments corporels ne peuvent que réduire le nombre de délinquants en Afrique du Sud (Van der Spuy et al. 2000: 173). Face à une campagne

European Journal of Turkish Studies, 15 | 2012 
de presse internationale critiquant la maltraitance de l'enfance, le ministre de la Justice s'exclame au Parlement en 1959: 'Where are we in South Africa if a child can no longer even be given a hiding ?' (Fourchard 2009: 7). Tout mineur passant une première fois devant une cour de justice reçoit une correction de quatre ou cinq coups de fouets, avant d'être placé en institution en cas de récidive ${ }^{34}$. Dans les institutions pénales, où l'effectif des moins de 21 ans est multiplié par trois entre 1933 et 1962, les coups de fouets sont une pratique systématique ${ }^{35}$.

Le recours au fouet par les organisations locales demeure également fréquent, même s'il est impossible de le quantifier. Les membres africains des conseils consultatifs ont une vision singulière de la violence des townships: dans la majorité des cas, ils associent la criminalité à la dérive incontrôlée de la jeunesse et à son manque de respect envers les aînés. Très tôt, ils réclament de pouvoir corriger les enfants et les jeunes de leur quartier (Bonner mars 1995: 21 (1) 123). Sofasonke Mpanza, membre du conseil consultatif de Johannesburg, raconte ainsi que, dans les cours, 'il suffirait de donner douze coups de fouet sous les pieds des délinquants pour se débarrasser définitivement des tsotsis et des Russians à Johannesburg'. La plupart de ces organisations (cours de parents, Legkotla, Magkotla, Peacemakers) recourrent à la chicotte, comme le reconnaissent encore aujourd'hui leurs anciens membres: 'nous avions l'habitude de frapper avec des battes de cricket et des bâtons notamment sur les articulations des bras et des jambes'; 'une "bonne correction sanglante" était nécessaire pour les récidivistes'; 'la seule médecine pour les enfants ce sont les coups de sjambok; tu dois leur apprendre la loi'. Frapper les enfants fait partie d'une norme sociale et seul un milieu circonscrit d'éduqués condamne ces pratiques dans les années $1970^{36}$. A partir de 1976, les pratiques punitives se modifient essentiellement parce que le fouet change de mains. Dans les cours populaires, les punitions corporelles sont la règle (Schärf et al. 1990: 362), tout comme dans les comités de quartier et les comités de rue : plusieurs coups de sjambok sont administrés directement au domicile des délinquants qui volent dans le quartier ${ }^{37}$. Les groupes les plus structurés, comme les Russians, dont on a trop rapidement dit qu'ils étaient par principe hostiles à l'ANC, s'opposent surtout au bouleversement des rapports sociaux qu'introduisent les comités de rue: ils se déclarent ainsi hostiles au châtiment d'un père de famille ayant administré lui-même une correction à son fils ou à sa femme (Kynoch 2005: 150).

L'avènement de la démocratie en 1994, la diffusion de la rhétorique des droits de l'homme et le vote d'une nouvelle loi sur l'enfance en 2005 modifient sensiblement le répertoire discursif sur l'indispensable recours au fouet pour éduquer la jeunesse. Les anciens membres des comités de rue et les organisations civiques locales qui luttent contre la criminalité ont été sensibilisés aux méfaits des violences arbitraires soit au cours d'ateliers de formation, soit au sein de structures plus formelles comme le West Coast anti-Crime Forum, né en 1996 de la fusion de trente organisations communautaires de la province (Kinnes 2000: 22). Malgré ces initiatives, les plaintes rapportées à la presse locale contre les violences perpétrées par les NW à Mitchell Plains demeurent nombreuses. Il semble néanmoins que l'usage de la correction soit moins systématique qu'auparavant: devenue illégitime, elle se transforme et se fait plus discrète.

On peut émettre l'hypothèse que l'usage très sélectif de la fouille au corps est la poursuite sous une autre forme de l'usage immodéré de la chicotte de la période de l'apartheid. Ainsi, sur les cinq patrouilles de nuit effectuées entre février et août 2009 à Manenberg et dans différents quartiers de Mitchell's Plain, la fouille au corps est 
systématiquement pratiquée sur les jeunes croisés au hasard des rondes. Les adultes hommes et femmes y échappent en revanche presque à chaque fois. Sont recherchées en priorité des amphétamines (le tik), dont l'usage s'est répandu massivement au cours des cinq dernières années, mais aussi couteaux et tournevis, arme blanche et outil incontournable du braqueur de voiture. Les fouilles sont parfois musclées et, dans une minorité de cas, aboutissent à des violences légères ou graves lorsqu'une personne est prise en 'flagrant délit': plusieurs jeunes tentant d'échapper à un contrôle sont frappés au visage, un autre soupçonné de tentative de viol est passé à tabac par une bonne partie des membres du NW et du responsable du CPF avant d'être livré à la police, des autres, surpris avec des prostituées au bord de la plage, sont mis à nu devant la patrouille hilare. Les principaux responsables de ces organisations reconnaissent que les cibles des patrouilles sont en priorité les jeunes. Dans l'esprit de ces organisations et de leurs membres, les principaux délits qui structurent la criminalité des townships (usage de drogues, agressions violentes, viols, cambriolages ou braquages de voitures) sont de fait étroitement identifiés à la jeunesse: ce répertoire est en grande partie identique à celui mobilisé durant la période de l'apartheid. La structure même de ces organisations, composée presque exclusivement de pères et de mères de famille, $\mathrm{y}$ est pour beaucoup: le discours tenu est la volonté à la fois de limiter les usages de la drogue et de protéger les enfants du quartier. La solution au problème de la criminalité de la jeunesse passe donc nécessairement par son enfermement ou son contrôle. Les jeunes s'y soumettent; ils protestent rarement, se conformant à une pratique considérée comme légitime car véhiculée par toutes les institutions du quartier, par la police et par un enracinement historique de pratiques devenues normes sociales.

\section{L'économie du volontariat}

31 Tous les membres qui s'engagent dans ces organisations disent vouloir œuvrer à la lutte contre la 'menace criminelle'. Il ne faut pas négliger ce discours, systématiquement mis en avant, tant la violence des townships constitue un levier puissant de mobilisation. Mais si la question de la violence est souvent obsessionnelle dans les townships, elle n'est pas toujours une clé d'explication suffisante pour déterminer qui s'engage pour la combattre et pour quelles raisons. Les sources écrites sont pauvres en la matière, et seuls les entretiens et formes d'observation participante permettent de lever une partie du voile. Les ressorts de la mobilisation s'y révèlent cependant multiples et difficiles à hiérarchiser. La construction de réseaux de sociabilité, l'affirmation de formes locales d'autorité, l'accès à des ressources dans un environnement paupérisé sont autant de mobiles à croiser avec l'histoire individuelle et familiale des membres de ces organisations.

32 A l'évidence, participer à de telles organisations, c'est d'abord vouloir recréer une sociabilité qui passe par un réinvestissement des espaces publics, par un engagement avec ses voisins, son quartier et plus rarement avec la ville entière. On patrouille pour sortir le week-end, rencontrer des gens, discuter autour d'un feu, apprendre à connaître ses voisins. L'un des traits communs à toutes ces organisations depuis un demi-siècle est leur rapport au temps hebdomadaire: elles patrouillent le vendredi et le samedi soir alors que, le plus souvent en Afrique, les organisations de vigilantes imposent un couvre-feu toutes les nuits de la semaine. Etant donnée l'intense activité des commissariats de police sud-africains le week-end, il ne semble pas illogique que ces organisations concentrent leurs efforts sur ces deux nuits. Cette spécificité a 
évidemment à voir avec l'histoire industrielle de l'Afrique du Sud, le versement des salaires aux mineurs, ouvriers et fonctionnaires le vendredi après-midi, la mise en place progressive d'une dissociation assez nette entre temps de travail et temps de loisirs et la concentration progressive des distractions autour de la dépense et de la redistribution des salaires de la classe ouvrière. À cet égard, une partie importante de la sociabilité noire comme des activités des gangsters s'organise autour des shebeens les nuits de week-end: les deux sont de fait intimement liées ${ }^{38}$. Groupements de gangsters et d'autodéfense ont ainsi en commun de vouloir bâtir un espace public nocturne convivial où pouvoir consommer en sécurité. Les activités sociales routinières des Peacemakers dans les années 1970 ou des comités de rue à la fin des années 2000 se déroulent autour d'un feu, parfois d'un braai ${ }^{39}$, qui réunit les résidents de la rue ou du bloc, afin de créer un espace sûr et convivial. Les comités de rue rassemblent aussi parfois des personnes plus âgées (dans la tranche d'âge des 60-80 ans), qui boivent du thé et du café toute la nuit, rompant ainsi leur isolement et leur ennui. Dans de nombreux quartiers de Mitchell's Plain, les nouveaux membres du NW affirment que participer à des patrouilles leur ont permis de rencontrer des voisins et d'étendre ainsi leur réseau de connaissances, même si la convivialité du NW paraît parfois bien déprimante ${ }^{40}$. Rappelons une fois de plus que ces réseaux de sociabilité vicinale se construisent en opposition aux sociabilités des plus jeunes, les NW dispersant systématiquement l'attroupement de ces derniers sur la voie publique.

Ce faisant, ces organisations participent de la redéfinition de l'autorité à l'échelle du quartier. Une parcelle de pouvoir est légalement conférée à chacun de leurs membres: celui d'arrêter, de fouiller et de remettre à la police les réfractaires à un ordre défini par les adultes et auquel les jeunes ont intérêt à se conformer pour éviter la garde-àvue. Les positions d'autorité dans ces organisations ne se fondent cependant ni sur l'âge, ni sur le sexe - sans doute parce que l'immense majorité de leurs membres sont des pères et mères de famille dans la tranche des 40-60 ans -, mais s'acquièrent en premier lieu par la longue expérience de terrain et en second par l'engagement politique ou la position sociale. Les responsables de NW sont des personnages connus à l'échelle du quartier. Ce sont des militants de longue date: plusieurs se disent actifs depuis plus de vingt ans. Cet engagement leur a permis de bâtir des réseaux d'interconnaissance locale qui sont éventuellement mobilisables pour d'autres tâches plus rémunératrices (service d'ordre de soirées privées, participation rémunérée à des évènements culturels par exemple). L'autorité du responsable se manifeste sur le terrain par de nombreux signes distinctifs: il assure le lien avec le commissariat, porte la radio prêtée par la police, revêt toujours un des signes distinctifs d'appartenance au groupe (le tee-shirt, le blouson de l'association locale), suggère un itinéraire au fur et à mesure de la soirée, met parfois à disposition sa voiture dans les quartiers plus aisés. Le fonctionnement des NW reste néanmoins souple, et chaque membre est investi d'une microparcelle d'autorité autorisant les comportements les plus variables, de la fouille musclée à la discussion amicale avec les interpellés.

Dans les quartiers les plus pauvres, participer à une organisation est également un moyen d'accéder à des ressources matérielles ou relationnelles. Selon les programmes de soutien gouvernementaux, les membres des NW reçoivent des bicyclettes, des blousons, des tee-shirts, des radios. Le nombre de Bambanani indemnisés par le gouvernement provincial varie également en fonction des quartiers. Ces ressources, apparemment dérisoires, semblent essentielles aux résidents des quartiers qui ne bénéficient pas de ces avantages ${ }^{41}$. Etre membre volontaire de l'ONG locale et puissante 
de Manenberg, c'est profiter de ses connexions avec le gouvernement. Soixantequatorze femmes de ce quartier ont ainsi été recrutées en 2005 par cette ONG en tant que Bambanani du programme Safer School du gouvernement provincial. Les cinquante rands journaliers d'indemnité ne sont pas suffisants pour nourrir une famille, certes, mais ils permettent à leurs bénéficiaires, des femmes très largement, de s'occuper de leurs enfants à la fin de leur journée de travail ou à la sortie des classes. Ce volontariat exclusivement féminin s'inscrit dans une économie politique locale marquée par la progression massive du chômage dans des quartiers qui ne bénéficient plus, depuis la fin de l'apartheid, de la Coloured Labour Preference Policy ${ }^{42}$. Nombre de ces femmes bénévoles sont de fait d'anciennes ouvrières au chômage. Leur engagement s'ancre dans une histoire familiale et dans une économie domestique locale particulière caractérisée par l'ampleur des violences conjugales. Beaucoup ont grandi dans un environnement de tradition policière, leur père ou leur frère ayant été membre des Peacemakers, du Pagad ou des NW. La plupart sont des mères isolées, ayant rejeté - ou ayant été délaissées par - un mari violent ou absent. Le souci prioritaire de ces mères de famille est de protéger leurs enfants de la violence de la rue. Ce faisant, elles construisent des formes de respectabilité qu'elles pensent être des modèles alternatifs à la respectabilité des chefs de gang et qui sont bien souvent les seuls modèles pour les jeunes garçons de ces quartiers (Jensen 2008; Salo 2004).

\section{La bureaucratisation de l'engagement volontaire}

Passée la période de mobilisation, la routine use cependant rapidement les énergies. Comme patrouiller est dangereux, fatigant et contraignant, ces patrouilles suivent des cycles de mobilisation et de démobilisation qui n'ont rien de particulier ni à l'Afrique du Sud, ni à l'Afrique en général. Les responsables des ONG des CPF et de la police savent qu'il est nécessaire de maintenir l'engagement des volontaires au-delà des périodes de mobilisation initiale s'ils veulent compter sur ces bonnes volontés non rémunérées. Pour cela, ils misent largement sur une culture du volontariat qui, pour l'essentiel, remonte à la période de l'apartheid: un engagement politique antérieur à 1994 s'est transmué depuis en engagement pour les causes les plus variées ${ }^{43}$. Des campagnes de mobilisation sont lancées à intervalle régulier sur les sujets les plus divers (manifestations contre la violence et la drogue, pétitions contre la libération sous caution des gangsters, journées sans violence domestique, journées des enfants sans violence, etc.). Les responsables d'ONG, de CPF et de la police sont alors transformés en chefs de service, organisant réunion sur réunion, au cours desquelles des objectifs sont assignés à chaque groupe de volontaires pour une période généralement courte. Par exemple, pour susciter l'émulation, le CPF de Mitchell's Plain a imposé à tous ses volontaires une culture du résultat: tous les trois mois, les représentants des douze secteurs de la zone sont invités à annoncer officiellement le nombre de nouveaux comités de rue, le nombre de nouveaux membres de NW et le nombre de volontaires recrutés comme réservistes ${ }^{44}$. Les volontaires se soumettent à ces exigences bureaucratiques. La lassitude existe, mais lorsqu'elle est exprimée en public - bien rarement au demeurant -, elle ne fait pas long feu.

36 Le volontariat est central dans la formation historique de l'Etat en Afrique du Sud, notamment grâce à un dispositif spécifique de la police sud-africaine. En 1960, la proclamation de l'état d'urgence à la suite de l'interdiction de l'ANC et du Parti communiste incite le gouvernement à mettre sur pied des comités de volontaires 
chargés de défendre la République sud-africaine contre les 'attaques terroristes'. Cette nouvelle force républicaine et citoyenne (bientôt connue sous le nom de 'réserve') doit assurer gratuitement le travail de la police prise par d'autres tâches. Elle s'ouvre dans les années 1960 aux communautés coloured et noires, notamment pour patrouiller dans les townships, une disposition reprise par le gouvernement ANC depuis $1994^{45}$. Ce dispositif n'est pas propre à l'Afrique du Sud, puisqu'on le retrouve dans de nombreux autres pays anglophones. En Afrique du Sud, néanmoins, les mobilisations contre le gangstérisme ont constitué, par-delà la rupture de la fin de l'apartheid, un moyen important pour la police de recruter des réservistes: les comités de lutte contre la délinquance des années 1960, les Peacemakers dans les années 1970, les NW dès les années 1980, les Bambanani depuis 2002 et les comités de rue depuis 2007 ont tous fourni de nombreux réservistes à la police nationale.

\section{Conclusion}

37 Les mobilisations contre le gangstérisme ont été réprimées lorsqu'elles ont été perçues comme hostiles au gouvernement en place. Elles ont surtout été le moyen pour l'Etat de recruter des volontaires pour accomplir le travail que la police ne pouvait assurer au quotidien, exemple relativement classique de formation de l'Etat en Afrique. La répression et le contrôle policiers sont historiquement très puissants en Afrique du Sud, et aujourd'hui, en partie grâce aux réservistes, le ratio de policiers par SudAfricain est l'un des plus élevés au monde ${ }^{46}$. Cette armada sud-africaine ne sert plus, comme au temps de l'apartheid, à réprimer les opposants politiques. Force d'encadrement exceptionnelle dans les zones riches, elle s'appuie dans les zones pauvres sur des dizaines de milliers de réservistes et de volontaires. L'engagement de ceux-ci ne peut néanmoins pas se réduire à leur 'capture' par l'Etat. Les acteurs rusent avec les dispositifs; ils ne perçoivent pas nécessairement le caractère intrinsèquement politique de ces initiatives, peuvent les considérer le cas échéant comme une ressource potentielle, alors que les plus familiers se rendent compte de leur faible degré d'innovation, voire dans certains cas de leur relative inefficacité.

Ce volontariat qui perdure depuis un demi-siècle sous les formes les plus variées ne se limite donc pas à la lutte contre la criminalité; ses membres sont au demeurant bien désarmés pour faire face à la violence des gangs, aux réseaux de drogue ou à la criminalité organisée. Toutes ces organisations d'aînés ont en commun, par-delà leur histoire cyclique, de construire un rapport d'autorité entre les parents et ceux qui ne le sont pas, de produire un ordre local, soit une communauté morale en lutte contre les désordres sociaux de la vie des townships. Ceux-ci sont identifiés depuis plusieurs décennies à un moment précis de la semaine (les soirs de week-end) et à un certain nombre d'activités légales mais suspectes (traîner dans la rue, boire au shebeen, fréquenter des prostituées, sortir seul avec une fille). Un tel ordre moral se construit aux dépens de la jeunesse, cible principale de la coercition, qui témoigne d'une continuité des pratiques que la période de lutte contre l'apartheid des années 1980 n'a finalement pas remis en question. Les jeunes se soumettent à un contrôle qui serait inimaginable ailleurs, tandis que les parents répondent aux injonctions des autorités et des voisins de les voir consacrer davantage de temps à surveiller leur rue ou leur quartier. Béatrice Hibou remarque à propos de la Tunisie qu'au-delà de la répression et du contrôle policiers les dispositifs de l'exercice du pouvoir façonnent les modalités de 
l'obéissance, voire de l'adhésion (ibid.). Au-delà de la répression policière en Afrique du Sud, ces mobilisations manifestent aussi un autre exemple de servitude volontaire.

\section{BIBLIOGRAPHIE}

Badenhorst, Cécile et Mather, Charles (1997) ‘Tribal recreation and recreating tribalism: culture, leisure and social control on South Africa's gold mines, 1940-1950', Journal of Southern African Studies, 23 (1), pp. 473-489.

Baker, Bruce (2002) 'Living with non-state policing in South Africa; the issues and dilemmas', Journal of Modern African Studies, 41 (1), p. 36.

Baker, Bruce (2008) Multi-Choice Policing in Africa, Uppsala, Nordiska Afrikainstitutet.

Bayart, Jean-François (2008) 'Hégémonie et coercition en Afrique subsaharienne. La "politique de la chicotte"', Politique africaine, no 110, pp. 123-152.

Bénit, Claire; Fourchard, Laurent et Wafer, Alex (2009) 'La circulation des initiatives sécuritaires collectives dans l'espace et dans le temps à Johannesburg', in C. Bénit-Gbaffou, S. Fabiyi et E. Peyroux (dir.), La Sécurisation des quartiers dans les villes africaines. Quels défis pour la gouvernance urbaine?, Paris et Johannesburg, Karthala et Ifas, pp. 115-141.

Bonner, Philip (1995) 'African urbanistion on the Rand between the 1930s and 1960s: its social character and political consequences', Journal of Southern African Studies, 21 (1), p. 126.

Botha, Anneli (2001) The Prime Suspects? The Metamorphosis of Pagad, Monograph 63, ISS.

Burger, Johan (2007) Strategic Perspectives on Crime and Policing in South Africa, Pretoria, Van Shaik Publishers, pp. 106-108.

Dixon, Bill et al. (2001) Gangs, Pagad and the State: Vigilantism and Revenge Violence in the Western Cape, Violence and Transition Series, vol. 2, Centre for the Study of Violence and Reconciliation.

Dooling, Wayne (2007) Slavery, Emancipation and Colonial Rule in South Africa, Scottsville, University of KwaZulu-Natal Press, pp. 84-89.

Dubresson Alain et al. (eds) (2008) Le Cap après l'apartheid. Gouvernance métropolitaine et changement urbain, Paris Karthala, p. 14.

Fourchard, Laurent (2006) 'Les territoires de la criminalité à Lagos et à Ibadan depuis les années 1930', Revue Tiers-Monde, no 185, janvier-mars pp. 91-107.

Fourchard, Laurent (2008) 'A new name for an old practice: vigilante in South-Western Nigeria', Africa, 78, 1, pp. 16-4.

Fourchard, Laurent (2009a) 'The making of the juvenile delinquent in Nigeria and South Africa, 1930-1970', History Compass, 7, p. 7.

Fourchard, Laurent (2009b) “'Shebeens”, sociabilité et pouvoir en Afrique du Sud au Xx siècle', in L. Fourchard, O. Goerg et M. Gomez-Perez, Lieux de sociabilité urbaine en Afrique, Paris, L'Harmattan, pp. 203-224. 
Galant, Raashied et al. (1996) Drugs, Gang, People's Power: Exploring the Pagad Phenomenon, Cape Town, Claremont Masjid.

Glaser, Clive (1998) 'We must infiltrate the Tsotsis: school politics and youth gangs in Soweto, 1968-1976', JSAS, 24 (2), p. 321.

Glaser, Clive (2000) Bo-Tsotsi: the Youth Gangs of Soweto, 1935-1976, (Social History of Africa), Portsmouth, NH, Oxford et Cape Town, Heinemann, James Currey et David Philip.

Goodhew, David (1993) 'The people’s police force: communal policing initiatives in the Western areas of Johannesburg, circa 1930-1962', Journal of Southern African Studies, 19 (3), pp. 447-470.

Hibou, Béatrice (2006) La Force de l'obéissance. Economie politique de la répression en Tunisie, Paris, La Découverte, pp. 95-96.

Jensen, Steffen (2008) Gangs, Politics and Dignity in Cape Town, Oxford, Johannesburg et Chicago, James Currey, University of Witwatersrand Press et University of Chicago Press.

Kelly, J. E. (2009) 'It is because of our Islam that we are there' the call of Islam in the United Democratic Front Era', African Historical Review, 41 (1), p. 126.

Kinnes, Irvin (2000) From Urban Street Gangs to Criminal Empires: the Changing Face of Gangs in the Western Cape, Monograph 48, ISS, Pretoria, p. 22.

Kynoch, Gary (2003) 'Friend or foe? A world view of community-police relations in Gauteng townships, 1947-1977', Canadian Journal of African Studies, 37, pp. 298-327.

Kynoch, Gary (2005) We Are Fighting the World: a History of the Marashea Gangs in South Africa, 19471999, Athens, Ohio University Press.

Kynoch, Gary (2009) ‘Urban violence in colonial Africa, Journal of Southern African Studies, 35, pp. 629-645.

Mistry, Duxita (1997) A Review of Community Policing, Johannesburg, Centre for the Study of Violence and Reconciliation.

National Archives South Africa (NASA), BAO 520/400, Memorandum on the need of establishment and/or recognition of village guards, submitted to the ministers of Justice and Native Affairs by the Johannesburg Advisory Board, 23 février 1951.

Penn, Nigel (1999) Rogues, Rebels and Runaways. Eighteenth-Century Cape Characters, Cape Town, David Philip, p. 79.

Pinnock, Don (1984) The Brotherhood: Street Gangs and State Control in Cape Town, Cape Town, Philip David.

Pratten, David et al. (eds) (2007) Global Vigilantes, Londres, Hurst \& Co.

Rauch, Janine et al. (2006) Police Reform in Post-conflict Africa. A Report, Cape Town, Institute for Democracy in South Africa (IDASA).

Salo, Elaine (2004) Respectable Mothers, Tough Men and Good Daughters: Producing Persons in Manenberg Township, South Africa, PhD thesis, Emory University.

Schärf, Wilfried et B. Ngocokoto (1990) «Images of punihsment in the people's courts of Cape Town, 1985-1987: from prefigurative justice to populist violence », in C. Manganyi et A. du Toit, Political Violence and the Struggle in South Africa, Londres, McMillan, p. 362.

Seekings, Jeremy (2001) 'Social ordering and control in the African townships of South Africa : an historical overview of extra-state initiatives from the 1940s to the 1990s', in W. Schärf et D. Nina 
(eds), The Other Law: Non-State Ordering in South Africa, Lansdowne, Cape Town, Juta \& Co., pp. 71-97.

Shaw, Mark (2002) Crime and Policing in Post Apartheid South Africa: Transformation Under Fire, Londres, Hurst \& Co., p. 98.

Shearing, Clifford et al. (2006) 'South Africa', in T. Jones et T. Newburn (eds), Plural Policing. A Comparative Perspective. Oxon, Routledge.

Singh, Anne-Marie (2008) Policing and Crime Control in Post Apartheid South Africa, Ashgate, Aldershot.

Steinberg, Jonny (2004) The Number, Cape Town, Jonathan Ball Publishers.

Steinberg, Jonny (2008a) 'Crime', in N. Sheperd et S. Robins (eds), New South African Keywords, Athens, Ohio University Press, p. 29.

Steinberg, Jonny (2008b) Thin Blue: the Unwritten Rules of Policing in South Africa, Johannesburg et Cape Town, Jonathan Ball Publishers.

Suttner, Raymond (2008) The ANC Underground in South Africa to 1976: a Social and Historical Studies, Johannesburg, Jacana Media.

Van der Spuy, Elrena, Schärf, Wilfried et Lever, Jeffrey (2000) 'The politics of youth crime and justice in South Africa, in C. Summer (ed.), The Blackwell Companion to Criminology, Oxford, Blackwell, p. 173.

Van Onselen, Charles (1982) New Babylon, New Nineveh: Everyday Life on the Witwatersrand, 1886-1914, Johannesburg, Ravan Press.

Van Onselen, Charles (2007) The Fox and the Flies: the Secret Life of a Grotesque Master Criminal, New York, Walker \& Company.

Waller, Richard (2006) 'Rebellious youth in colonial Africa', Journal of African History, 47, p. 85

Western Cape Provincial Government, Bambanani Against Crime, Cape Town, 2007.

\section{NOTES}

1. Concernant les criminels transnationaux, les gangs de tsotsis et de Russians à Johannesburg et dans les villes minières du Rand, voir Kynoch 2005; Glaser 2000; Van Onselen 1982 et 2007. Dans la région du Cap, les gangs sont souvent liés au système pénitentiaire, mais leur historicité est encore peu étudiée. Voir Jensen 2008; Steinberg 2004; Pinnock 1984. Ces recherches n'ont pour l'instant pas d'équivalent dans l'historiographie de l'Afrique subsaharienne. Voir Waller 2006: 85.

2. Selon Jonny Steinberg, 85 des crimes violents aujourd'hui se déroulent pendant le week-end, $80 \%$ des agressions et des attaques à main armée ont lieu dans un rayon de 500 mètres autour d'un shebeen (débit de boisson) et la plupart des victimes et des agresseurs sont saouls au moment des faits. Steinberg 2008a: 29.

3. L'essor du 'vigilantisme' en Afrique est ainsi souvent associé au déclin de l'Etat postcolonial ou aux 'transitions démocratiques'. Retracer les filiations historiques des groupes de vigilantes montre au contraire que ceux-ci procèdent de l'Etat colonial et postcolonial. Voir Fourchard 2008: 78 (1) 16-40. Pour une vision mondiale du phénomène, voir Pratten et al. 2007.

4. Les relations toujours conflictuelles et difficiles entre la police et les townships noirs sont d'après Steinberg héritées des pratiques policières de l'apartheid. Steinberg $2008 \mathrm{~b}$. 
5. Le régime de l'apartheid a classé la population sud-africaine en quatre groupes: les Noirs, les Coloured, les Indiens et les Blancs. Ces catégories sont d'un usage commun aujourd'hui.

6. NASA Memorandum on the need..., doc. cit.

7. Stirtonville (1956), Chiawelo (fin des années 1950 début des années 1960), Zola (1960-1965), Orlando (1965), Mofolo (1965-1967), Natalspruit (1955, puis de nouveau en 1969), Springs (1969) et Tladi/Moletsane (1969-1972). Voir Glaser 2004; 'Boardman hits at ban guards move', World, 15 janvier 1969; 'Kwa Thema key rapist strikes again twice', World, 7 janvier 1969.

8. Des pratiques d'autodéfense existaient également à District Six, mais nous sommes peu informés sur leur mode de fonctionnement. Don Pinnock évoque cependant une organisation du nom de Globe qui se criminalisa dans les années 1950. Voir Pinnock 1984: 24-29.

9. Living in Manenberg and Bonteheuwel, workshop sur les conditions de vie dans plusieurs townships coloured de Cape Flats et leurs implications sur la fréquence des comportements déviants, National Institute for Crime Prevention and the Rehabilitation of Offenders, Cape Town, 1975. Entretien avec Aefan Davids, ancien Peacemaker responsable de cours, février 2009, Manenberg, Cape Town. Entretien avec Robin Roberts, ancien Peacemaker, mai 2009, Manenberg, Cape Town. 10. Entretien avec Robin Roberts, ancien Peacemaker, mai 2009, Manenberg, Cape Town.

11. Living in Manenberg..., doc. cit.

12. 'Crime watch for "plain”, Plainsman, 15 juillet 1987; 'Crime watches under spotlight', Plainsman, 19 août 1987.

13. Ibid., pp. 37-49.

14. Ibid., p. 50.

15. Entretien, Department of Community Safety, août 2009, Cape Town.

16. L'ANC a gouverné la province du Cap entre 1994 et 1998, puis ce fut le tour du Parti national entre 1998 et 2001, avant que celle-ci ne passe à une coalition du New National Party et de l'ANC (de 2001 à 2004) puis à l'ANC (2004-2009). Pendant cette décennie, un nouveau parti, l'Alliance démocratique (DA), né de la fusion du Parti démocratique (DP) et du NNP en 2000, n'a cessé d'accroître son influence dans la région. La DA a pu gouverner la métropole du Cap entre 2000 et 2001, municipalité qui est passée ensuite à une coalition ANC/NP avant de repasser à une coalition dirigée par la DA aux élections locales de 2006. Elle a remporté les élections provinciales dans le Western Cape en 2009, seule province qui échappe à l'ANC en Afrique du Sud à ce jour.

17. Au recensement de 2001, les coloured de la métropole du Cap représentaient $46 \%$ du total, les Africains noirs $31,2 \%$, les Blancs $21,2 \%$ et les Indiens asiatiques 1,5\%. Voir Dubresson et al. (eds) 2008:14.

18. Western Cape Provincial Government, Bambanani Against Crime, Cape Town, 2007.

19. En 2007, le gouvernement se vantait d'avoir entraîné 1000 volontaires.

20. Mitchell's Plain (un million d'habitants) n'a que 17 établissement scolaires inclus dans le programme, alors que Manenberg, dix fois moins peuplée, a vu toutes ses écoles incluses dans le programme, soit 14 établissements, dont 11 écoles primaires considérées comme peu dangereuses. Cela est lié pour l'essentiel à la présence à Manenberg d'une ONG puissante tenue par des leaders de l'ANC.

21. Avec un million d'habitants, Mitchell's Plain est la principale zone résidentielle coloured de toute l'Afrique du Sud. Elle est constituée de quartiers pauvres et middle class qui votent en grande majorité pour la DA.

22. 'Rocklands residents stand together against drug peddling', Plainsman, 28 janvier 2009.

23. En 2009, sur les vingt comités de rue que j'ai visités dans trois quartiers de Mitchell's Plain, dix-huit conservaient cette fonction, deux surveillaient des shebeens.

24. http://www.saps.gov.za/_dynamicModules/internetSite/HPProvNews.asp?PID=9

25. 'Mbeki and Zuma call for street committees', Cape Argus, 5 avril 2008. 
26. Integrated Regional Information Networks (IRIN), South Africa: Government Resurrecting Street Committees, 24 september 2008: http://www.unhcr.org/refworld/docid/48e085e41e.html [13 november 2009]

27. 'Enquiry to probe Zille allegations', Plainsman, 3 octobre 2007, p. 1; 'I am proud of where I come from. Director Vearey', Plainsman, 3 octobre 2007, p. 6.

28. Ibid.

29. 'Un équipement inadéquat de la voirie en caméras, un manque d'éclairage public, le pouvoir d'évincer des locataires dealers de drogue très peu utilisé.' Voir 'Enquiry to probe Zille allegations', art. cit.

30. Entretien avec le directeur de la police communautaire du gouvernement provincial, Le Cap, août 2009.

31. Entretien avec Fazlin Abel, janvier 2009, Manenberg.

32. Constat fait à multiples reprises par plusieurs intervenants au cours de la conférence History and Legacy of Punishment in Southern Africa, Université d'Oxford, mars 2010.

33. Constat avancé pour la période antérieure avant 1960 par Kynoch 2009: pp.629-645. Ce constat repose peut-être sur la faiblesse des travaux disponibles sur l'histoire de la criminalité violente en Afrique. Celle-ci était déjà présente dans les quartiers pauvres des villes du sud-ouest du Nigeria depuis les années 1930, même si les niveaux de violence étaient probablement moindres. Voir Fourchard 2006: 185 91-107.

34. Débats parlementaires, Hansard, 12 mai 1959 (col. 5696).

35. Passant de 21526 détenus de moins de 21 ans en 1933 à 70000 en 1962: République d'Afrique du Sud, Bureau de la statistique, Statistics of Offences and of Penal Institutions, 1949-1962.

36. Perceptible par exemple dans le journal World.

37. Entretien avec Tembile Ndabeni, mars 2009, Cape Town.

38. Par exemple, dans les régions minières du Witwatersrand, les gangs des Russians proposent aux mineurs sotho de prendre en charge la sécurité et l'organisation de leur week-end en leur fournissant un ensemble de services (mise à disposition de shebeens et de prostituées, accompagnement sécurisé du shebeen à la mine). Voir Kynoch 2005. Sur les shebeens comme activité structurante de la sociabilité noire en Afrique du Sud, voir Fourchard 2009: 203-224.

39. Barbecue en afrikaans.

40. Boire un café à la machine automatique de la seule station d'essence du quartier ouverte la nuit.

41. Entretiens avec différents membres du quartier de Heidevel qui jalousent les avantages accordés au quartier voisin de Manenberg, décembre 2009. Je remercie Chloé Buire de m'avoir fait rencontrer certains des membres actifs de ce quartier.

42. Politique officielle du gouvernement à partir de 1954 qui visait à réserver les emplois dans la province du Cap occidental aux Coloured plutôt qu'aux Africains. Cette politique a été abolie par le nouveau gouvernement de l'ANC en 1994.

43. Lutte contre le sida, lutte contre le gangstérisme, soutien aux victimes de violences, aide aux personnes âgées ou aux orphelins, aide logistique pour les évènements sportifs mondiaux comme la Coupe du monde de foot de 2010.

44. Réunion du CPF de Mitchell's Plain, juin 2009, Le Cap.

45. En 1994, la police sud-africaine comptait 115000 membres pour 70000 réservistes et exmembres. En 2006, 156000 membres et 30000 réservistes.

46. Avec 200000 policiers et réservistes pour 50 millions d'habitants, il est de $1 / 250$, soit un ratio plus élevé que celui de tous les pays d'Europe. Si l'on ajoute les 307000 agents du secteur de la sécurité privée, ce ratio s'élève à $1 / 100$, soit celui du régime répressif de la Tunisie de Ben Ali. Voir Hibou 2006: 95-96. 


\section{RÉSUMÉS}

Les mobilisations populaires contre le gangstérisme sont au cœur de la vie sociale et politique des townships sud-africains depuis des décennies mais leur histoire particulière s'inscrit mal dans un récit historique qui verrait une police de l'apartheid politisée, militarisée, répressive et hostile aux populations non blanches laisser progressivement la place à une police moins répressive, moins politisée, plus coopérative avec les populations 'historiquement défavorisées' depuis la fin du régime de l'apartheid en 1994. A partir d'archives, d'entretiens avec les leaders locaux et des personnels de police et des pratiques d'observation participante au sein de patrouilles de quartiers pauvres du Cap, cet article entreprend de retracer les trajectoires de ces organisations de lutte contre la criminalité et leur rapport ambivalent à l'institution policière -de la coopération à la répression - avant, pendant et après la période d'apartheid. Il dévoile la multiplicité des ressorts de la mobilisation : de l'engagement partisan, au besoin de sociabilité et au désir d'Etat et de mise en ordre du quartier. Il rend aussi compte de la violence interne de ces organisations qui participent sur la longue durée à la production d'un ordre social coercitif, mis en place par les parents et ainés principalement contre la jeunesse, perçue comme dangereuse ou en danger. En produisant une communauté morale en lutte contre les désordres sociaux des townships, ces volontaires luttent in fine moins contre la violence réelle des gangsters et dealers de drogue qu'ils ne reproduisent cette fracture générationnelle héritée de la période de l'apartheid.

Popular mobilizations against gangsters have been central in the social and political life of South African townships for decades. Yet this specific history does not tie in well with a narrative that would highlight a radical change from a politicized, militarized and repressive apartheid police, with a strong hostility to non-white populations, to a police less repressive, less politicized and more friendly with 'historically disadvantaged' people since the end of apartheid in 1994. This article draws on archival work, interviews with local leaders and police staff, participant observation in neighborhood watch patrols in poor neighborhoods of Cape Town, so as to understand the history of crime-fighting organizations and their relationship with the police from cooperation to repression - before, during and after the period of apartheid. It reveals how these forms of mobilizations have been permeated by a multiplicity of individual choices: from partisan politics to the need for sociability, from a demand for more state intervention to the wish to police the neighborhood. It also reflects the inside violence of the organizations which participate in the production of a coercive social order, as implemented by parents and elders who perceived the youth as dangerous or as being in danger. These volunteers thus produced a moral community which, while fighting against the violence of gangsters and drug dealers in the townships, ultimately reproduced the generational gap inherited from the apartheid era.

\section{INDEX}

Mots-clés : violences, sécurité, coercition, communauté morale, mobilisation

Keywords : violence, security, coercion, moral community, mobilization 


\section{AUTEUR}

\section{LAURENT FOURCHARD}

Les Afriques dans le monde (LAM), Institut d'études politiques de Bordeaux, Fondation nationale des sciences politiques (FNSP) 1.fourchard@sciencespobordeaux.fr 